Portland State University

PDXScholar

Electrical and Computer Engineering Faculty

Publications and Presentations

8-1-1981

\title{
Electromagnetic modes of an inhomogeneous sphere
}

Lee W. Casperson

Portland State University

Follow this and additional works at: https://pdxscholar.library.pdx.edu/ece_fac

Part of the Electrical and Computer Engineering Commons

Let us know how access to this document benefits you.

\section{Citation Details}

Lee W. Casperson. Electromagnetic modes of an inhomogeneous sphere. Applied Optics, Volume 20, Number 15 (August 1981), pp. 2738-2741.

This Article is brought to you for free and open access. It has been accepted for inclusion in Electrical and Computer Engineering Faculty Publications and Presentations by an authorized administrator of PDXScholar. Please contact us if we can make this document more accessible: pdxscholar@pdx.edu. 


\title{
Electromagnetic modes of an inhomogeneous sphere
}

\author{
Lee W. Casperson
}

\begin{abstract}
In many cases the electromagnetic modes of a sphere can be obtained exactly. Special simplifications occur when the conductivity, complex permittivity, and complex permeability vary only in the radial direction, and static and time dependent examples are discussed. With quadratic or Coulomblike permittivity variations, the wave functions resemble the quantum mechanical harmonic oscillator or hydrogen atom wave functions, and similar angular momentum operators are obtained.
\end{abstract}

\section{Introduction}

Several of the more important and more difficult problems of electromagnetic theory involve the propagation characteristics of inhomogeneous media. Sometimes the media in question can be assumed to have a basic spherical symmetry, and nonspherical media are often approximated by spheres. Two recent studies were concerned with the surface modes of inhomogeneous spheres, and the results were related to the waveguiding atmospheres of stars and planets. ${ }^{1,2}$ Another class of problems involves electromagnetic modes which penetrate throughout an inhomogeneous sphere, and it is this class that is emphasized in the present study. It should be possible to fabricate such spheres using, for example, techniques that have been developed for graded-index fibers. If the media have gain, resonant laser modes may result, ${ }^{3}$ depending on the level of radiation losses. The electromagnetic wave functions of a sphere are similar to the corresponding wave functions of quantum mechanics, and the angular momentum operators for the two cases are essentially identical.

In Sec. II, Maxwell's equations are reduced to a set of ordinary differential equations from which the field components can be derived. In this treatment the azimuthal field variations are governed by the familiar spherical harmonics. The radial equation for situations involving radial variations of the complex permittivity and slow time variations is solved in Sec. III, and the angular momenta of the modes are derived in the Appendix.
The author is with University of California, School of Engineering \& Applied Science, Los Angeles, California 90024.

Received 19 December 1980.

0003-6935/81/152738-04\$00.50/0.

(C) 1981 Optical Society of America.

\section{Field Equations}

Maxwell's equations for the electromagnetic field vectors can be written

$$
\begin{aligned}
& \nabla \times \mathbf{E}=-\mu_{0} \frac{\partial \mathbf{H}}{\partial t}-\mu_{0} \frac{\partial \mathbf{M}}{\partial t}, \\
& \nabla \times \mathbf{H}=\epsilon_{0} \frac{\partial \mathbf{E}}{\partial t}+\frac{\partial \mathbf{P}}{\partial t}+\sigma \mathbf{E} .
\end{aligned}
$$

The fields of interest in this study are nearly harmonic in time, and it is useful to introduce the substitutions

$$
\begin{aligned}
\mathbf{E} & =\operatorname{Re}^{\prime} \exp (i \omega t), \\
\mathbf{H} & =\operatorname{ReH}^{\prime} \exp (i \omega t), \\
\mathbf{P} & =\operatorname{Re}^{\prime} \exp (i \omega t), \\
\mathbf{M} & =\operatorname{Re}^{\prime} \mathbf{M}^{\prime} \exp (i \omega t),
\end{aligned}
$$

where the primed quantities are the complex amplitudes of the unprimed quantities. With these substitutions Eqs. (1) and (2) become

$$
\begin{aligned}
& \nabla \times \mathbf{E}^{\prime}=-\mu_{0}\left(\frac{\partial \mathbf{H}^{\prime}}{\partial t}+i \omega \mathbf{H}^{\prime}\right)-\mu_{0}\left(\frac{\partial \mathbf{M}^{\prime}}{\partial t}+i \omega \mathbf{M}^{\prime}\right), \\
& \nabla \times \mathbf{H}^{\prime}=\epsilon_{0}\left(\frac{\partial \mathbf{E}^{\prime}}{\partial t}+i \omega \mathbf{E}^{\prime}\right)+\left(\frac{\partial \mathbf{P}^{\prime}}{\partial t}+i \omega \mathbf{P}^{\prime}\right)+\sigma E^{\prime} .
\end{aligned}
$$

At a later point attention will be restricted to separable time and space dependences, and it would be possible at the outset to assume a simple complex frequency and time independent field amplitudes. Retaining the time derivatives does, however, lead to a useful analogy with Schroedinger's equation as discussed below.

The advantage of this complex notation is that inphase and out-of-phase components of the polarization and magnetization can be represented by simple complex susceptibilities. Thus, Eqs. (7) and (8) reduce to

$$
\begin{aligned}
& \nabla \times \mathbf{E}^{\prime}=-\mu_{0}\left(1+\chi_{m}\right)\left(\frac{\partial \mathbf{H}^{\prime}}{\partial t}+i \omega \mathbf{H}^{\prime}\right), \\
& \nabla \times \mathbf{H}^{\prime}=\epsilon_{0}\left(1+\chi_{e}\right) \frac{\partial \mathbf{E}^{\prime}}{\partial t}+i \omega \epsilon_{0}\left(1+\chi_{e}+\frac{\sigma}{i \omega \epsilon_{0}}\right) \mathbf{E}^{\prime},
\end{aligned}
$$


where $\chi_{m}$ and $\chi_{e}$ are, respectively, the complex magnetic and electric susceptibilities.

Equations (9) and (10) may be combined to obtain a single equation for the electric field amplitude

$$
\begin{aligned}
\nabla \times \nabla \times \mathbf{E}^{\prime}= & -\mu_{0}\left(1+\chi_{m}\right)\left[\epsilon_{0}\left(1+\chi_{e}\right) \frac{\partial^{2} \mathbf{E}^{\prime}}{\partial t^{2}}\right. \\
& +2 i \omega \epsilon_{0}\left(1+\chi_{e}-\frac{i \sigma}{2 \omega \epsilon_{0}}\right) \frac{\partial \mathbf{E}^{\prime}}{\partial t} \\
& \left.-\omega^{2} \epsilon_{0}\left(1+\chi_{e}-\frac{i \sigma}{\omega \epsilon_{0}}\right) \mathbf{E}^{\prime}\right]+\frac{\nabla \chi_{m} \times \nabla \times \mathbf{E}^{\prime}}{1+\chi_{m}} .
\end{aligned}
$$

If the electric field amplitude varies slowly compared with an optical cycle, the second time derivative of $\mathbf{E}^{\prime}$ can be neglected. It is also convenient to introduce the complex permeability $\mu=\mu_{0}\left(1+\chi_{m}\right)$ and the complex permittivities $\epsilon=\epsilon_{0}\left(1+\chi_{e}-i \sigma / \omega \epsilon_{0}\right)$ and $\epsilon^{\prime}=\epsilon_{0}\left(1+\chi_{e}\right.$ $\left.-i \sigma / 2 \omega \epsilon_{0}\right)$. With these substitutions Eq. (11) reduces to

$$
\nabla \times \nabla \times \mathbf{E}^{\prime}=-2 i \omega \mu \epsilon^{\prime} \frac{\partial \mathbf{E}^{\prime}}{\partial t}+\omega^{2} \mu \epsilon \mathbf{E}^{\prime}+\frac{\nabla \mu}{\mu} \times \nabla \times \mathbf{E}^{\prime} .
$$

For the problem of interest here the complex permeability and permittivity vary only in the radial direction. Thus the vector wave equation can be reduced to a scalar wave equation by means of the substitution $^{4}$

$$
\mathbf{E}^{\prime}(r, \theta, \phi, t)=\nabla \times\left[\mathbf{i}_{r} r \psi(r, \theta, \phi, t)\right] .
$$

With this substitution Eq. (12) is replaced by the single scalar equation

$$
\nabla^{2} \psi=\omega^{2} \mu \epsilon \psi+2 i \omega \mu \epsilon^{\prime} \frac{\partial \psi}{\partial t}+\frac{1}{r \mu} \frac{d \mu}{d r} \frac{\partial(r \psi)}{\partial r} .
$$

This result can be separated by means of the usual substitution $\psi(r, \theta, \phi, t)=R(r, t) Y_{l}^{m}(\theta, \phi, t)$, and the radial equation is

$$
\begin{aligned}
\frac{1}{r} \frac{\partial^{2}}{\partial r^{2}}(r R)-\frac{1}{r \mu} \frac{d \mu}{d r} \frac{\partial(r R)}{\partial r}-2 i \omega \mu \epsilon^{\prime} & \frac{\partial R}{\partial t} \\
& +\left[\omega^{2} \mu \epsilon-\frac{l(l+1)}{r^{2}}\right] R=0 .
\end{aligned}
$$

Some typical solutions of this equation are discussed in the following section. Besides these modes, a similar alternative set can be obtained by starting with the wave equation for the magnetic field and using the substitution $\mathbf{H}^{\prime}=\nabla \times\left[\mathbf{i}_{r} r \psi(r, \theta, \phi, t)\right]$ in place of Eqs. (12) and (13).

\section{Specific Radial Profiles}

In principle Eq. (12) could be used to describe field distributions which have shapes and amplitudes that are functions of time. The results would be exact if the second time derivative had been retained. The only solutions considered here are those in which the time and space dependences are separable. Thus it is assumed that the radial function can be written in the form

$$
R(r, t)=A(r) \exp (s t) .
$$

With this substitution Eq. (15) can be written

$$
\frac{1}{r} \frac{\partial^{2}}{\partial r^{2}}(r A)-\frac{1}{r \mu} \frac{d \mu}{d r} \frac{\partial r A}{\partial r}+\omega^{2} \mu \epsilon\left(\frac{1-2 i s \epsilon^{\prime}}{\omega \epsilon}\right) A-\frac{l(l+1)}{r^{2}} A=0 .
$$

Since the dielectric relaxation time will usually be long compared with an optical cycle, $\epsilon^{\prime}$ can for simplicity be replaced by $\epsilon$ in the small $s / \omega$ term. The result can be written

$$
\frac{1}{r} \frac{\partial^{2}}{\partial r^{2}}(r A)+k^{2} A-\frac{l(l+1)}{r^{2}} A=0,
$$

where $\mu$ is independent of $r$, and $k^{2}$ is defined by

$$
k^{2}=\omega^{2} \mu \epsilon(1-2 i s / \omega) .
$$

It is now necessary to specify the radial dependence of coefficient $k$. One interesting special case occurs when $k$ is a quadratic function of radius. Much progress has been made in the fabrication of optical fibers having quadratic-index variations, and it is reasonable to suppose that similar profiles could be achieved in spherical systems. Also quadratic-index media are the most familiar analytically of the various possible waveguiding systems. Thus it is assumed here that parameter $k$ has the usual weak quadratic profile ${ }^{5}$ :

$$
k=k_{0}-k_{2} r^{2} / 2 \text {. }
$$

The real and imaginary parts of $k_{0}$ and $k_{2}$ follow from Eq. (19), and Eq. (18) becomes

$$
\frac{d^{2} A}{d r^{2}}+\frac{2}{r} \frac{d A}{d r}+k_{0}^{2} A-k_{0} k_{2} r^{2} A-\frac{l(l+1)}{r^{2}} A=0 .
$$

As with other quadratic guiding media, it is helpful to first factor out a solution of the form ${ }^{5}$

$$
A(r)=B(r) \exp \left(-i Q r^{2} / 2\right) .
$$

With this substitution Eq. (21) can be separated into the two equations

$$
\begin{aligned}
\frac{d^{2} B}{d r^{2}}+\left(\frac{2}{r}-2 i Q r\right) \frac{d B}{d r}-3 i Q B+k_{0}^{2} B-\frac{l(l+1)}{r^{2}} B & =0 .
\end{aligned}
$$

The solutions of Eq. (23) are

$$
Q= \pm i\left(k_{0} k_{2}\right)^{1 / 2} .
$$

Equation (24) can be simplified by means of the substitution

$$
B(r)=C(r) r^{l},
$$

and the result is

$$
\frac{d^{2} C}{d r^{2}}+\left[\frac{2(l+1)}{r}-2 i Q r\right] \frac{d C}{d r}+\left[k_{0}^{2}-i Q(2 l+3)\right] C=0 .
$$

The solutions to Eq. (27) can be written in two somewhat different forms depending on whether the propagation constant $k$ can be assumed to be real. ${ }^{6}$ In the simplest cases $k$ is essentially real, $Q$ from Eq. (25) is imaginary, and the requirement that $C$ be real leads to the equation

$$
\frac{d^{2} C}{d r^{2}}+\left[\frac{2(l+1)}{r}+2 Q_{i r}\right] \frac{d C}{d r}+\left[k_{0}^{2}+Q_{i}(2 l+3)\right] C=0,
$$

where $Q_{i}$ denotes the imaginary part of $Q$. A useful 


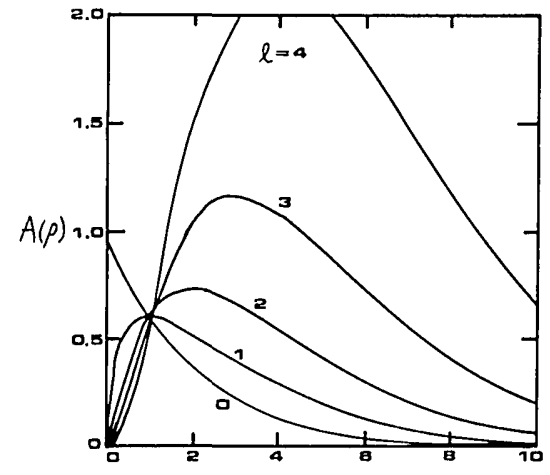

$\rho$

Fig. 1. Radial field variations of a quadratically profiled sphere as a function of the radial coordinate $\rho=-Q_{i} r^{2}$ for various values of the index $l$. Index $p$ is equal to zero for these examples.

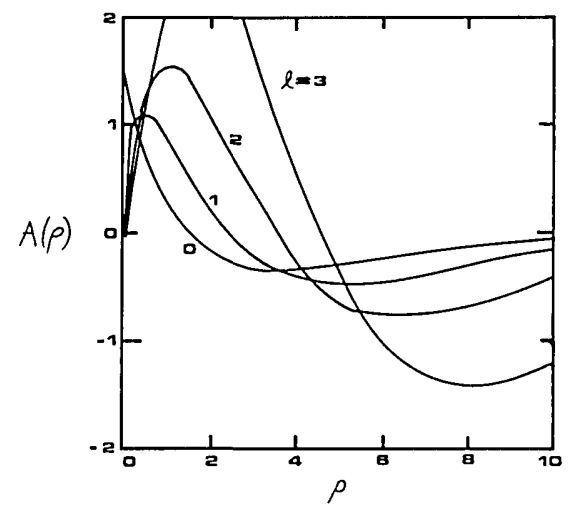

Fig. 2. Radial field variations of a quadratically profiled sphere with the index $p=1$.

change of variables is $\rho=-Q_{i} r^{2}$, and with this substitution Eq. (28) reduces to

$$
\rho \frac{d^{2} C}{d \rho^{2}}+\left[l+\frac{3}{2}-\rho\right] \frac{d C}{d \rho}+p C=0,
$$

where parameter $p$ is given by

$$
p=-\frac{k_{0}^{2}+Q_{i}(2 l+3)}{4 Q_{i}} .
$$

But Eq. (29) is a standard form of the Laguerre differential equation, and the solution can be written in terms of the associated Laguerre functions ${ }^{7}$

$$
C(\rho)=L_{p}^{l+1 / 2}(\rho) .
$$

In the somewhat more complicated case where $Q$ is complex, the solutions to Eq. (27) can be expressed in terms of Laguerre functions of complex argument.

By combining Eqs. (22), (26), and (31), the radial part of the wave function can be written

$$
A(\rho)=\rho^{l / 2} \exp (-\rho / 2) L_{p}^{l+1 / 2}(\rho),
$$

where there has been a slight renormalization of the factor in front. This equation is plotted in Figs. 1 and 2 for various values of indices $l$ and $p$. The fields move outward as $l$ increases and develop more complicated radial variations with increasing values of $p$. The actual vector field components can be obtained from Eq. (13), and the angular momentum of these modes is discussed in the Appendix. Similar procedures can be used to obtain the wave functions for other power law radial gain or refraction profiles. ${ }^{8}$ Thus, for a $r^{-1 / 2}$ radial variation of the index of refraction the propagation constant can be written

$$
k=a r^{-1 / 2},
$$

and the radial equation becomes

$$
\frac{d^{2} A}{d r^{2}}+\frac{2}{r} \frac{d A}{d r}+\frac{a^{2}}{r} A-\frac{l(l+1) A}{r^{2}}=0 .
$$

But this is similar to the radial equation for the wave functions of a particle in a Coulomb potential, and the solutions are well known. ${ }^{9}$

\section{Conclusion}

In this study several aspects of the electromagnetic modes of an inhomogeneous sphere have been considered in detail. The analysis has included radial variations of the conductivity and the in-phase and out-ofphase components of polarization and magnetization. Complete solutions have been obtained for the case in which the radial profiles are quadratic, and the approach for other profiles is similar. Quadratic profiles are of special interest because of their analytical and fabricational similarities to the graded-index media developed for fiber-optic applications. Other profile types might be relevant to the modes of waveguiding atmospheres or gravitational lenses.

The author is pleased to acknowledge the assistance of S. Kalson in checking the calculations.

\section{Appendix: Angular Momentum}

It has already been remarked that there are close similarities between the electromagnetic wave functions of a radially inhomogeneous sphere and the quantum mechanical wave functions corresponding to a spherically symmetric potential. In this section the mechanical property angular momentum is examined for the electromagnetic modes of a sphere. As a starting point it is noted that the Poynting vector for an electromagnetic field can be written

$$
\mathbf{S}=\mathbf{E} \times \mathbf{H} \text {. }
$$

If the field is nearly harmonic in time, the average Poynting vector is

$$
\mathbf{S}_{\mathrm{av}}=\frac{1}{2} \operatorname{Re}\left(\mathbf{E}^{\prime} \times \mathbf{H}^{\prime *}\right),
$$

where $\mathbf{E}^{\prime}$ and $\mathbf{H}^{\prime}$ are again the complex field amplitudes. Finally, the average momentum density carried by the fields is related to the Poynting vector by ${ }^{10}$

$$
\mathbf{p}_{\mathrm{av}}=\mathbf{S}_{\mathrm{av}} / \mathrm{c}^{2} \text {, }
$$

where $c$ is the speed of light. These formulas are directly applicable to the present problem. 
The electric field components of a harmonic wave are given by Eq. (13), and from Eq. (9) the harmonically varying magnetic field can be written

$$
\mathbf{H}^{\prime}(r, \theta, \phi)=\frac{i}{\omega \mu} \nabla \times \mathbf{E}^{\prime}(r, \theta, \phi) .
$$

Therefore, the angular momentum density for $\mu$ real is

$$
\begin{aligned}
\mathbf{L} & =\mathbf{r} \times \mathbf{p}_{\mathbf{a v}}=\frac{1}{2 c^{2}} \operatorname{Re}\left[\mathbf{r} \times\left(\mathbf{E} \times \mathbf{H}^{*}\right)\right] \\
& =-\frac{1}{2 \omega \mu c^{2}} \operatorname{Re}\left\{\left[\nabla \times\left(\mathbf{i}_{r} r \psi\right)\right]\left[\mathbf{r} \cdot \nabla \times \nabla \times\left(\mathbf{i}_{r} r \psi^{*}\right)\right]\right\} \\
& =-\frac{l(l+1)}{2 \omega \mu c^{2}} \operatorname{Im}\left[\psi^{*}(\overline{\mathbf{r}} \times \nabla) \psi\right],
\end{aligned}
$$

where use has been made of the vector identity

$$
\overline{\mathbf{r}} \cdot \nabla \times \nabla \times(\mathbf{r} \psi)=\frac{r \partial^{2}}{\partial r^{2}}(r \psi)-r^{2} \nabla^{2} \psi=l(l+1) \psi .
$$

By this point the analogy with the ordinary quantum mechanical angular momentum density operator is becoming conspicuous. This analogy can be heightened by writing Eq. (A5) in the form

$$
\overline{\mathbf{L}}=\alpha \operatorname{Re}\left\{\psi^{*}[\mathbf{r} \times(i \hbar \nabla)] \psi\right\}
$$

The factor in brackets is the complex conjugate of the ordinary quantum mechanical angular momentum ${ }^{11}$ operator, and the parameter $\alpha$ is given by

$$
\alpha=\frac{l(l+1)}{2 \omega \mu c^{2} h} .
$$

Since the normalization of the wave functions is still arbitrary, there is no significance to the numerical value of $\alpha$. For wave functions of the type derived previously it follows from Eq. (A6) that the angular momentum density can be written more simply as

$$
\mathbf{L}=-\alpha \mathbf{i}_{\theta} \frac{m \hbar}{\sin \theta} \psi^{*} \psi
$$

The total $z$ component of angular momentum is obtained by integrating over all space according to

$$
\begin{aligned}
L_{z} & =\iiint \frac{-\alpha m \hbar}{\sin \theta} \psi^{*} \psi \overline{\mathbf{i}}_{\theta} \cdot \overline{\mathbf{i}}_{z} r^{2} \sin \theta d r d \theta d \phi \\
& =\alpha m \hbar \iiint \psi^{*} \psi r^{2} \sin \theta d r d \theta d \phi .
\end{aligned}
$$

For a suitable normalization this could be the usual result $L_{z}=m \hbar$. Thus the $z$ component of the angular momentum of the electromagnetic wave functions is essentially identical to the expectation value of the corresponding quantum mechanical angular momentum, and related observations apply to the angular momentum carried by multipole radiation fields. ${ }^{12}$

A similar result can be obtained for 1-D propagation problems. Thus for an $x$-polarized plane wave propagating in the $z$ direction, the electric field vector can be written

$$
\mathbf{E}^{\prime}=\mathbf{i}_{x} \psi(z),
$$

and the corresponding magnetic field is

$$
\mathbf{H}^{\prime}=\frac{i}{\omega \mu} \nabla \times \mathbf{E}^{\prime} .
$$

Therefore, with Eqs. (A1)-(A3) the momentum density carried by the wave can be written

$$
\overline{\mathbf{P}}_{\mathrm{av}}=-\left(2 \omega \mu c^{2}\right)^{-1} \mathbf{i}_{z} \operatorname{Im}\left(\psi^{*} \nabla \psi\right)=\alpha \mathbf{i}_{z} \operatorname{Re}\left[\psi^{*}(i \hbar \nabla) \psi\right],
$$

where $\alpha$ is now given by

$$
\alpha=\left(2 \omega \mu c^{2} \hbar\right)^{-1} .
$$

Thus the plane wave momentum in a volume of space also resembles the expectation value of the corresponding quantum mechanical momentum operator.

Finally, it may be noted that there is a close similarity between the quantum mechanical wave equation and the electromagnetic scalar wave equation. In particular, for a nonconducting medium with constant permeability, Eq. (14) reduces to

$$
\nabla^{2} \psi+\omega^{2} \mu \epsilon \psi=2 i \omega \mu \epsilon \frac{\partial \psi}{\partial t} .
$$

With substitutions $c^{2}=(\mu \epsilon)^{-1}$ and $\hbar \omega=m c^{2}$, this is the complex conjugate of Schrödinger's equation for an electron in a uniform potential equal to minus one half of the rest energy

$$
\frac{-\hbar^{2}}{2 m} \nabla^{2} \psi-\frac{m c^{2}}{2} \psi=-i \hbar \frac{\partial \psi}{\partial t} .
$$

It should be noted, however, that the more exact electromagnetic equation for the wave function $\psi$ [Eq. (11)] includes a second time derivative, which would not always be negligible. In quantum mechanics physically interesting quantities like momentum or angular momentum are obtained by calculating the expectation value of certain postulated operators. In electromagnetics, on the other hand, some of the same basic forms are obtained without new postulates or probability interpretations.

\section{References}

1. L. W. Casperson, J. Opt. Soc. Am. 65, 399 (1975).

2. L. W. Casperson, Astrophys. Space Sci. 48, 389 (1977).

3. C. G. B. Garrett, W. Kaiser, and W. L. Bond, Phys. Rev. 124, 1807 (1961).

4. J. A. Stratton, Electromagnetic Theory (McGraw-Hill, New York, 1941), p. 415.

5. H. W. Kogelnik and T. Li, Appl. Opt. 5, 1550 (1966).

6. L. W. Casperson, J. Opt. Soc. Am. 66, 1373 (1976).

7. M. Abramowitz and I. A. Stegun, Eds., Handbook of Mathematical Functions, NBS (U.S. GPO, Washington, D.C., 1964; Dover, New York, 1965), p. 781.

8. P. M. Morse and H. Feshbach, Methods of Theoretical Physics (McGraw-Hill, New York, 1953), p. 1670.

9. Ref. 8, p. 1663.

10. Ref. 4, p. 158.

11. L. I. Schiff, Quantum Mechanics (McGraw-Hill, New York, 1968), p. 82.

12. See, for example, J. D. Jackson, Classical Electrodynamics (Wiley, New York, 1962), Sec. 16.3. 